\title{
Standing wave integrated Fourier transform spectrometer for imaging spectrometry in the near infrared
}

\author{
Gaël D. Osowiecki*a , Mohammad Madi ${ }^{\mathrm{b}}$, Ivan Shorubalko ${ }^{\mathrm{c}}$, Irène Philipoussis ${ }^{\mathrm{a}}$, Edoardo Alberti ${ }^{\mathrm{b}}$, \\ Toralf Scharf ${ }^{\mathrm{a}}$, Hans Peter Herzig ${ }^{\mathrm{a}}$, \\ ${ }^{a}$ Optics \& Photonics Technology Laboratory, Institute of Microengineeering (IMT), École \\ Polytechnique Fédérale de Lausanne (EPFL), Rue de la Maladière 71b, 2002 Neuchâtel,

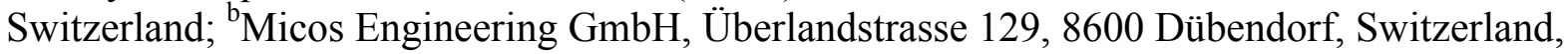 \\ ${ }^{\circ}$ Laboratory for Reliability Science and Technology, EMPA, Swiss Federal Laboratories for \\ Materials Science and Technology, 8600 Dübendorf, Switzerland
}

\begin{abstract}
We show the miniaturization and parallelization of a scanning standing wave spectrometer with a long term goal of creating a compact imaging spectrometer. In our standing wave integrated Fourier transform spectrometer, light is injected with micro-lenses into several optical polymer waveguides. A piezo actuated mirror located at the waveguide end-facet can shift the interferogram to increase its sampling frequency. The spatial distribution of the standing wave intensity inside the waveguide is partially scattered out of the plane by a periodic metallic grating and recorded by a CCD camera. We present spectra acquisition for six adjacent waveguides simultaneously at a wavelength of $632.8 \mathrm{~nm}$.
\end{abstract}

Keywords: Imaging spectrometer, SWIFTS, Waveguide, Microlens, Lippmann interferogram, Scattering

\section{INTRODUCTION}

Imaging spectrometry is a way of taking pictures with a lot more information than detected in the usual visible wavelength range that our eyes can see. Extending the information that each pixel can record in the ultra-violet (UV) or infrared (IR) region can reveal new information of great interest about the type of material which is present in the image. Imaging spectrometers are mostly used for remote sensing applications. The National Aeronautics and Space Administration (NASA) and the European Space Agency (ESA) have developed several imaging spectrometer like ${ }^{1-3}$ : "Landsat-8", "AVIRIS", "Hyperion", "ALICE", "MERIS" and "VIRTIS" to cite only some of them, having different missions of inspecting the broad electro-magnetic spectrum from UV to IR. Most of these imaging spectrometers are taking a lot of space and load, which results in expensive loads in satellites to be launched in space.

Here we want to present a new concept for a miniaturized scanning imaging spectrometers based on a standing wave integrated Fourier transform spectrometer (SWIFTS) ${ }^{4,5}$. SWIFTS spectrometers are based on the recording of a standing wave created by the interference of two waves. The first attempts to record such a standing wave where made by Gabriel Lippmann who won the Nobel Prize in 1908. He used a thin photographic emulsion with sub-wavelength silver halide grains laying on top of a liquid mercury mirror to permanently record the standing waves created by the different wavelengths of the light coming from a scene ${ }^{6,7}$. Here we will present a way to record the interferogram in a nonpermanent way. This will be done by dividing an image into individual pixels. Each pixel will be composed by a waveguide in which a standing wave will be created by a mirror at its end. This standing wave will be acquired by probing the evanescent field. Because of several technological limitations, the standing wave intensity will be locally under sampled, enabling a bandwidth limited to few nanometers. This limitation can be overcome by replacing the fixed mirror with a scanning mirror. By shifting the position of the mirror that back reflects the incoming wave, the interferogram is physically moved with respect to the scattering samplers, thus leading to an increase of the interferogram sampling ${ }^{8}$. The Fourier transform of the interferogram will then result in the light spectrum corresponding to the initial unknown light injected into the waveguides.

\section{PRINCIPLE OF LIPPMANN SWIFTS IMAGING SPECTROMETER}

A standing wave inside a waveguide can be created in two different manners. Either by the superposition of two counter propagating waves or by only one wave which is back reflected by a mirror at the end of the waveguide. We will use the second configuration, which is called Lippmann configuration. In the latter, the optical path difference is equal to zero at

Imaging Spectrometry XX, edited by Thomas S. Pagano, John F. Silny, Proc. of SPIE Vol. 9611, 96110P ( 2015 SPIE · CCC code: 0277-786X/15/\$18 · doi: 10.1117/12.2188170 
the mirror surface. Thus if a broad spectrum is injected in the waveguide the center of the interferogram is located at the mirror surface and quickly decays. The spatial sampling of the interferogram reflects in the Fourier domain as the attainable spectral bandwidth, while the length of the waveguide reflects in the Fourier domain as the attainable spectral resolution. The main problem is the fact that it is difficult to extract the standing wave intensity with a spatial sampling frequency, which respects the Nyquist-Shannon sampling theorem. With currently available technologies, the attainable bandwidth is limited to few nanometers, while users are generally interested in adopting imaging spectrometers with larger bandwidth. In our case, we focused on the detection within the wavelength range from $750 \mathrm{~nm}$ to $775 \mathrm{~nm}$, with $0.12 \mathrm{~nm}$ spectral resolution, with system level requirements approaching those of an imaging spectrometer for atmospheric studies and operating in the Oxygen-A band. The spectral resolution defines the minimum length of sampling the signal. For $0.12 \mathrm{~nm}$ spectral resolution, at least a length of $1 \mathrm{~mm}$ must be sampled. For covering the bandwidth between $750 \mathrm{~nm}$ to $775 \mathrm{~nm}$, the period at which we should place the nanosamplers (array of antenna) to respect the minimal sampling frequency is $T=3.6 \mu \mathrm{m}$ according to equation 1 when the effective index of the waveguide is 1.575 . In equation 1 the effective refractive index is assumed to be constant through the bandwidth and should be taken at the central wavelength (i.e. $762.5 \mathrm{~nm}$ ). This means that in the case a line array camera is used to record the scattered light intensity its pixel size should be similar to this period. Our aim here is to extend the detected wavelength range of a single waveguide without being limited by the minimum available pixel size of commercially available line array CCDs.

$$
\mathrm{T}=\frac{\lambda_{\text {min }} \cdot \lambda_{\text {max }}}{4 \cdot n_{\text {eff }}\left(\lambda_{\text {max }}-\lambda_{\text {min }}\right)}
$$

This is done by using a moving mirror rather than a fixed mirror to shift the interferogram positon. The mirror, shown in figure 1, is moved by a piezo actuator in small nanometric steps for which an image is recorded each time. Thanks to this, we can merge the multiple images acquired during the mirror movement and achieve a much higher sampling frequency of the interferogram and thus extending the bandwidth of the spectrometer.

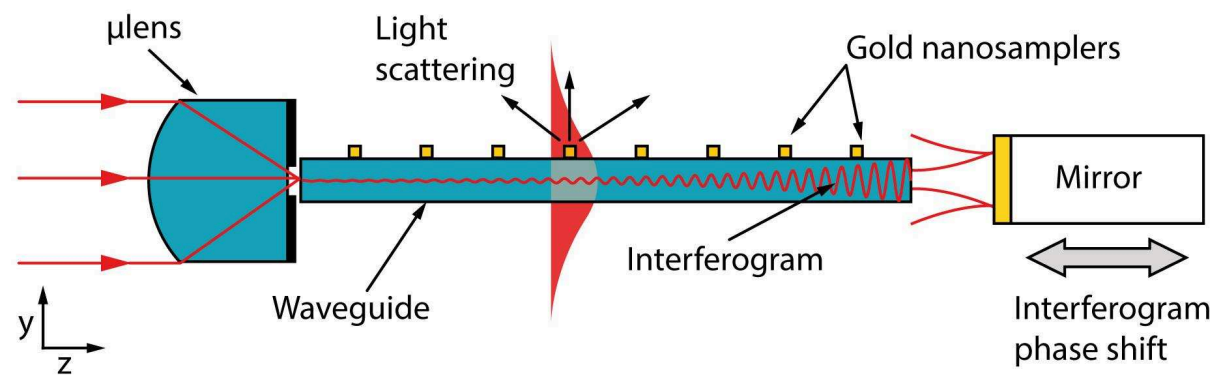

Figure 1. Sketch of the Lipmann SWIFTS configuration with a moving mirror. This allows to increase the sampling frequency of the interferogram, in other terms allow to acquire spectra with larger bandwidth with respect to the static configuration.

Nevertheless an issue with this approach is to couple enough light back into the waveguide after the reflection at the mirror. Because of diffraction, the divergence of the light beam exiting the waveguide facet leads to a loss of back coupling which is dependent of the mirror's position. That's why a careful calibration needs to be done for each mirror step.

\section{MULTIPLE WAVEGUIDE CONFIGURATION FOR IMAGING PURPOSES}

In the case of an imaging spectrometer using a push broom scanning configuration we need to use multiple adjacent waveguides to create a line array detector. In our bread boarding, the nanosamplers scattered light intensity is acquired through an imaging system and a CCD camera perpendicular to the waveguide plane. In our setup a field of view of 2 $\mathrm{mm}$ by $2 \mathrm{~mm}$ covers 6 adjacent waveguides while fulfilling required spectral resolution. In that case the alignment of the injection optics and the mirror becomes challenging with respect to that of a single waveguide case (figure 2). A microlens array is used in front of the waveguide facets to inject the light into each individual waveguide. To avoid any 
light injection into the cladding of the waveguides, chrome apertures of $10 \mu \mathrm{m}$ in diameter are located on the backside of the microlens array. The microlenses are focusing collimated light directly onto the aperture plane. A single piezo actuated gold mirror is used to reflect the light of all waveguides at the same time.

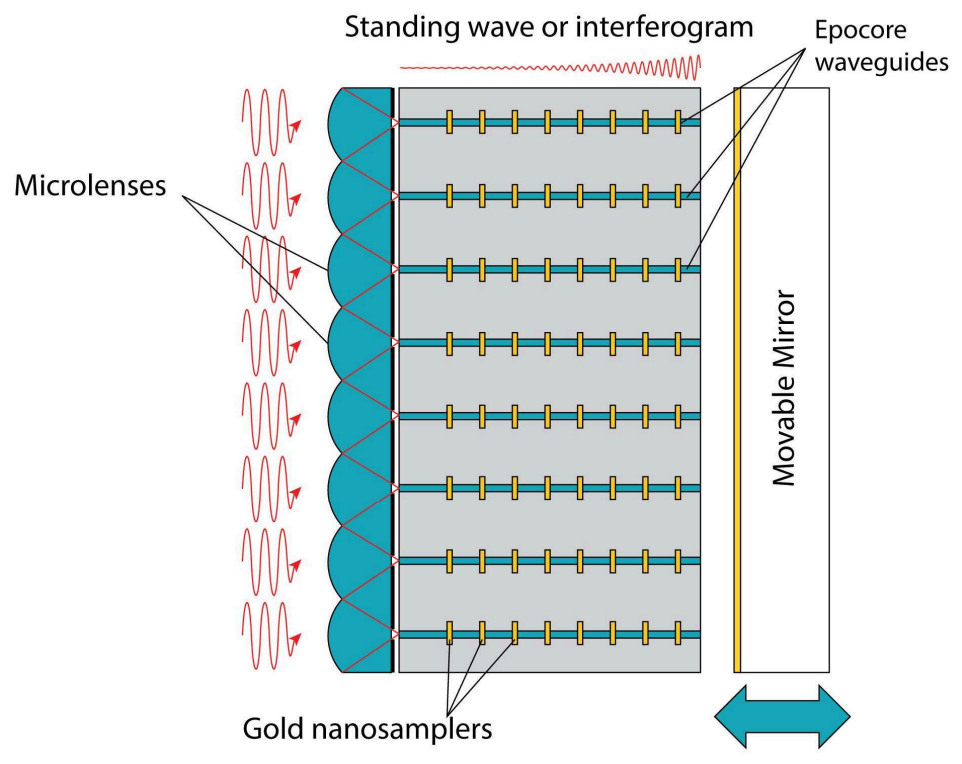

Figure 2. Sketch of the scanning imaging Lippmann SWIFTS configuration.

\section{FABRICATION OF THE SPECTROMETER}

The scanning imaging SWIFTS chip consists of the injection microlenses, the core waveguide chip with its gold nanosamplers and a custom made gold mirror. Here we quickly review the fabrication process of all these elements.

Microlenses are used to inject the light coming from the imaging system into the different waveguides ${ }^{9}$. These microlenses are fabricated on a glass substrate having an array of apertures of $10 \mu \mathrm{m}$ in diameter on the backside. These apertures are fabricated with a lift-off technique using a $150 \mathrm{~nm}$ chrome layer (10x the penetration depth at $800 \mathrm{~nm}$ ). Alignment marks are also present on the backside to be able to align each aperture to each individual microlens.

The microlenses are made out of AZ9260 photoresist having a refractive index of about $\mathrm{n}_{\text {lens }}=1.6$ at $800 \mathrm{~nm}$. The glass substrate is coated with a $600 \mathrm{~nm}$ thin base layer of AZ1518 which is then burned at $160^{\circ} \mathrm{C}$ for an hour. A top layer of AZ9260 having a thickness of $22 \mu \mathrm{m}$ is then coated on top of the base layer. This second layer is patterned with photolithography in cylinder shapes having a diameter of $245 \mu \mathrm{m}$. These cylinders are then molten above the glass transition temperature to be transformed into lenses at $150^{\circ} \mathrm{C}$. In a further step it is possible to etch them into glass, if needed. 

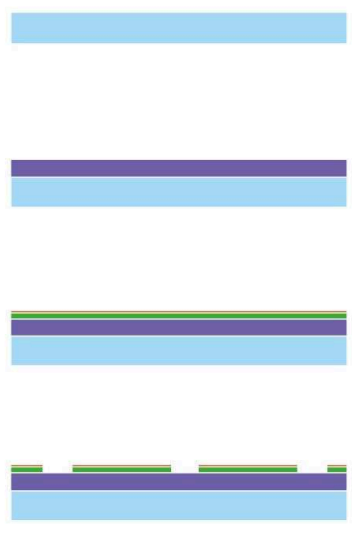

E-beam exposition, copper removal and developpement
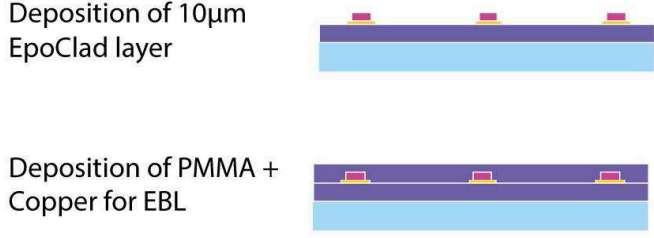

Deposition of $15 \mu \mathrm{m}$ upper EpoClad layer

Gluing of a $500 \mu \mathrm{m}$ thick glass wafer with NOA-61 glue

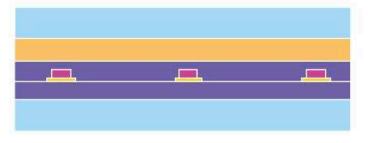

Patterning of the EpoCore waveguides aligned with gold nano-sampler

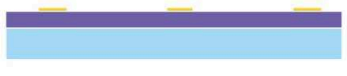

Gold deposition and

lift-off process

Figure 3: Sketch of the different fabrication steps of the waveguide chip of the SWIFTS spectrometer.

The waveguide chip is realized on a $500 \mu \mathrm{m}$ thick glass substrate. On top of the substrate, the waveguides and the cladding are made out of EpoClad and EpoCore epoxy-based UV photosensitive negative resist from Micro Resist Technology $\mathrm{GmbH}$. The first step consist to coat the glass with a $10 \mu \mathrm{m}$ thick EpoClad layer flowed by a flood exposure to be cross-linked. In a second step, the nano-samplers are patterned thanks to e-beam lithography. A $70 \mathrm{~nm}$ layer of PMMA is deposited on top of the EpoClad wafer. This is followed by an evaporation of $20 \mathrm{~nm}$ of copper to make the surface conductive and suitable for e-beam lithography. Nano-samplers having a size of $50 \mathrm{~nm}$ (width) x $20 \mathrm{~nm}$ (height) x $8 \mu \mathrm{m}$ (length) are patterned at $30 \mathrm{kV}$. After development of the exposed PMMA a $\mathrm{O}_{2}$ plasma descum is realized to clean the PMMA residues and a $20 \mathrm{~nm}$ gold layer is evaporated with a preliminary chrome flash for better adhesion. The PMMA is then lift-off in an acetone bath, which ends the process for the fabrication of the gold nano-samplers. The next step is to pattern the waveguide made out of EpoCore ${ }^{10}$. This is done by spin-coating a $2 \mu \mathrm{m}$ thick EpoCore layer on top of the nano-samplers, exposing it after precise alignment of the photolithographic mask with the nano-samplers and developing it. These waveguides are then covered by a spin-coated $15 \mu \mathrm{m}$ thick EpoClad cladding, which is cured by UV light. Because of further end-facet polishing problems a second glass substrate needs to be glued on top of the stack. This is done with a spin-coated layer of NOA-61 UV curing glue. Afterwards the wafers are diced into $5 \mathrm{~mm}$ x $10 \mathrm{~mm}$ chips.

\section{PRELIMINARY MEASUREMENTS}

The aim of our preliminary measurements is to acquire the spectrum of a helium-neon laser lasing at $632.8 \mathrm{~nm}$. The spectrum of the six adjacent waveguides has to be recorded simultaneously like showed in figure 4 . This has been done by using an SBIG ST-8300M cooled CCD camera with an inverted camera objective resulting in field of view of $2 \mathrm{~mm}$ by $2 \mathrm{~mm}$. The mirror was moved by an open-loop piezo having a maximum travel of $17.4 \mu \mathrm{m}$ with mirror steps of $1 \mu \mathrm{m}$. The EpoCore waveguides where designed to support a quasi-TE mode and a quasi-TM mode from $600 \mathrm{~nm}$ to $1000 \mathrm{~nm}$. The pitch of the nanosamplers is $10 \mu \mathrm{m}$. The distance between each waveguide is $250 \mu \mathrm{m}$. 


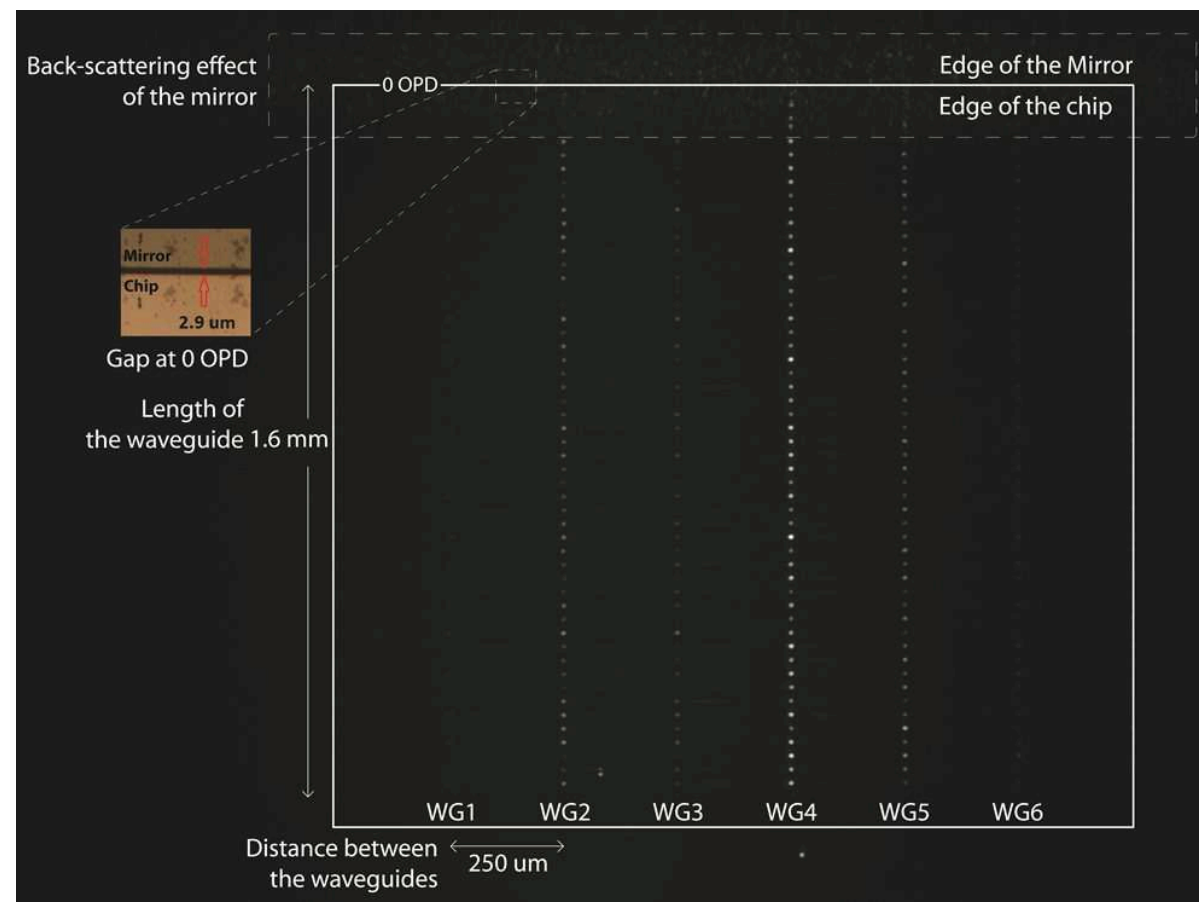

Figure 4: An acquired image of the six adjacent waveguides scattering the light injected from the HeNe laser.

The retrieved interferogram information has been treated in several steps prior to Fourier transfer operation. These steps include calibration of sampler's position, calibration of nano-samplers scattering efficiency, and the interferogram DCoffset removal which originates from the loss in the waveguide and the backscattering of nano-samplers that modifies the shape of interferogram. In figure 5(a) a spectrum including the actual technical limitations is simulated and compared with the experimental result shown in figure 5(b). The spectrum of helium-neon laser shown in figure 5(b) is reconstructed in undersampling conditions for a band-limited signal. 

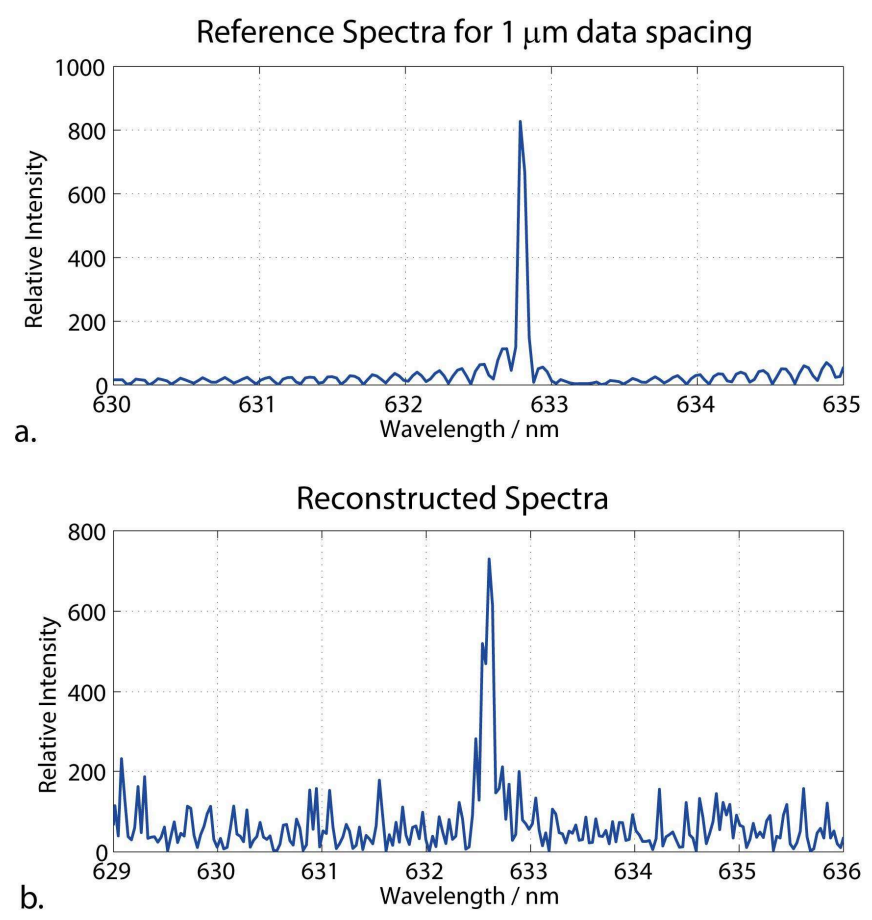

Figure 5: a) The spectra from a simulation in experimental conditions using a HeNe wavelength at $632.8 \mathrm{~nm}$. b) The reconstructed spectrum taken from the scattering of 200 nanosamplers and 15 different mirror position of $1 \mu \mathrm{m}$ steps (equivalent to a bandwidth of about $90 \mathrm{~nm}$ ).

\section{CONCLUSION}

The presented work shows a new approach in scanning imaging SWIFTS Lippmann spectrometers. The latter aims for an imaging spectrometer application by using a movable mirror to increase the bandwidth of a single waveguide by increasing the sampling of the standing wave. The fabrication process of epoxy based EpoCore waveguides showed the feasibility of reasonably large single mode waveguides with very low losses on the order of $0.3 \mathrm{~dB} / \mathrm{cm}$. We showed the first results of spectra of six adjacent waveguides extracted with a SWIFTS spectrometer using a movable mirror.

\section{ACKNOWLEDGEMENT}

This work is funded under ESA TRP Activity TEC-MMO/2011/143 aiming at design and development of Miniature High Performance Imaging Spectrometer for Remote Sensing.

\section{REFERENCES}

[1] Green, R. O., Eastwood, M. L., Sarture, C. M., Chrien, T. G., Aronsson, M., Chippendale, B. J., Faust, J. A., Pavri, B. E., Chovit, C. J., et al., "Imaging Spectroscopy and the Airborne Visible/Infrared Imaging Spectrometer (AVIRIS)," Remote Sens. Environ. 65(3), 227-248 (1998).

[2] Pearlman, J. S., Barry, P. S., Segal, C. C., Shepanski, J., Beiso, D.., Carman, S. L., "Hyperion, a space-based imaging spectrometer," IEEE Trans. Geosci. Remote Sens. 41(6), 1160-1173 (2003).

[3] Coradine, A., Capaccioni, F., Drossart, P., Semery, A., Arnold, G., Schade, U., Angrilli, F., Barucci, M. A., Bellucci, G., et al., "Virtis : an imaging spectrometer for the rosetta mission," Planet. Space Sci. 46, 1291-1304 (1998).

[4] le Coarer, E., Blaize, S., Benech, P., Stefanon, I., Morand, A., Lérondel, G., Leblond, G., Kern, P., Fedeli, J. M., et al., "Wavelength-scale stationary-wave integrated Fourier-transform spectrometry," Nat. Photonics 1(8), 473-478 (2007). 
[5] Bonneville, C., Thomas, F., de Mengin Poirier, M., Le Coarer, E., Benech, P., Gonthiez, T., Morand, A., Coutant, O., Morino, E., et al., "SWIFTS: a groundbreaking integrated technology for high-performance spectroscopy and optical sensors," Proc. SPIE 8616, 86160M-861615M (2013).

[6] Lippmann, G., "Sur la théorie de la photographie des couleurs simples et composées par la méthode interférentielle," J Phys Theor Appl 3(1), 97-107 (1894).

[7] Lippmann, G., "On Colour Photography by the Interferential Method," Proc. R. Soc. Lond. 60, 10-13 (1896).

[8] Malak, M., Jefimovs, K., Philipoussis, I., Di Francesco, J., Guldimann, B.., Scharf, T., "Functional micro-nano structures for on-chip fourier transform spectrometers," 2014 Symp. Des. Test Integr. Packag. MEMSMOEMS DTIP, 1-4 (2014).

[9] Nussbaum, P., Völkel, R., Herzig, H. P., Eisner, M.., Haselbeck, S., "Design, fabrication and testing of microlens arrays for sensors and microsystems," Pure Appl. Opt. J. Eur. Opt. Soc. Part A 6(6), 617 (1997).

[10] Malak, M., Philipoussis, I., Herzig, H. P.., Scharf, T., "Polymer based single mode optical waveguide for spectroscopy applications," Proc. SPIE 8846, 88460-884615 (2013). 